\title{
Prognostic Utility of Vitamin D in Acute Coronary Syndrome Patients in Coastal Norway
}

\author{
Patrycja A. Naesgaard, ${ }^{1,2}$ Volker Pönitz, ${ }^{1,2}$ Hildegunn Aarsetoey, \\ Trygve Brügger-Andersen, ${ }^{1,2}$ Heidi Grundt, ${ }^{2,3}$ William S. Harris, ${ }^{4}$ \\ Harry Staines, ${ }^{5}$ and Dennis W. T. Nilsen ${ }^{1,2}$ \\ ${ }^{1}$ Department of Cardiology, Stavanger University Hospital, Stavanger, Norway \\ ${ }^{2}$ Department of Clinical Science, University of Bergen, Bergen, Norway \\ ${ }^{3}$ Department of Medicine, Stavanger University Hospital, Stavanger, Norway \\ ${ }^{4}$ Department of Medicine, Sanford School of Medicine, University of South Dakota and OmegaQuant Analytics (LLC), \\ Sioux Falls, SD, USA \\ ${ }^{5}$ Sigma Statistical Services, Balmullo, UK
}

Correspondence should be addressed to Patrycja A. Naesgaard; patrycja_anna_szczepaniak@hotmail.com

Received 20 October 2014; Revised 1 January 2015; Accepted 5 January 2015

Academic Editor: Serge Masson

Copyright (C) 2015 Patrycja A. Naesgaard et al. This is an open access article distributed under the Creative Commons Attribution License, which permits unrestricted use, distribution, and reproduction in any medium, provided the original work is properly cited.

Background. An inverse relationship between cardiovascular risk and levels of vitamin D and omega-3 index may exist. Objectives. To evaluate the prognostic utility of serum 25-hydroxyvitamin D [25(OH)D] in 871 patients with suspected acute coronary syndrome (ACS) and to assess the seasonal correlation between $25(\mathrm{OH}) \mathrm{D}$ and the omega-3 index in 456 ACS patients from southwestern Norway. Results. In the univariate analysis the hazard ratio (HR) at 2-year follow-up for all-cause mortality in the highest as compared to the lowest quartile of $25(\mathrm{OH}) \mathrm{D}$ in the total population was 0.61 (95\% confidence interval (CI), 0.371.00), $P=0.050$. At 7-year follow-up, the corresponding HR for all-cause mortality was 0.66 ( $95 \% \mathrm{CI}, 0.49-0.90), P=0.008$, and for females alone 0.51 (95\% CI, 0.32-0.83), $P=0.006$. Quartile survival did not differ in the multivariable analysis, whereas $25(\mathrm{OH}) \mathrm{D}<40 \mathrm{nM}(<16 \mathrm{ng} / \mathrm{mL})$ was found to be independently related to mortality. Seasonal differences in $25(\mathrm{OH}) \mathrm{D}$, but not for the omega-3 index, were noted, and the two biomarkers were positively correlated, especially during winter-spring; Pearson's correlation coefficient was $0.358, P<0.001$. Conclusion. Vitamin D levels are related to survival, especially in females, and correlate with the omega-3 index.

\section{Introduction}

In observational studies vitamin $\mathrm{D}$ and $\mathrm{n}-3$ polyunsaturated fatty acids (PUFA) have been found to be inversely related to cardiovascular disease (CVD) $[1,2]$. Whether these factors are causal or are simply markers of a generally lower-risk lifestyle is still unsettled.

Low vitamin D levels have been associated with hypertension, obesity, dyslipidemia, and diabetes mellitus, reflecting an increased cardiovascular (CV) risk burden [3]. However, also high levels can be associated with increased total and $\mathrm{CV}$ mortality, as described by a U-shape or reverse J-curve [4-6].
N-3 fatty acids may exert beneficial effects on hemodynamics, lipid metabolism, inflammation, thrombosis, and ventricular arrhythmias [7]. Fish is the major food source of long-chain n-3 PUFA and includes eicosapentaenoic acid (EPA) and docosahexaenoic acid (DHA) [8]. The omega-3 index is a measure of these fatty acids, given as a percentage of total fatty acids in red blood cell membranes [9].

Vitamin D is mainly synthesized in the skin from 7dehydrocholesterol under the influence of sun exposure and the remaining $10-20 \%$ is ingested in the diet in which fatty fish is a major contributor [10]. The synthesis of vitamin D in response to ultraviolet $\mathrm{B}$ (UVB) radiation in sunlight depends 
on factors such as latitude, altitude, time of year and day-time, weather, age, skin pigmentation type, clothing, and sunscreen and is influenced by environment (pollution) and lifestyle [11]. Conditions such as poor nutrition and chronic illness are also associated with vitamin D deficiency [12].

The prognostic utility of vitamin $\mathrm{D}$ in relation to longterm CV outcomes has been evaluated in subjects without a history of CVD, as in the Framingham Offspring study [13] and in patients with established CVD $[14,15]$. We have previously shown that 2- and 5-year total and cardiac mortality is decreased in the highest as compared to the lowest quartile of vitamin $\mathrm{D}$ in a coronary chest-pain population living at altitudes exceeding 1000 meters in subtropical northern Argentina [16], and this relationship is stronger in females than in males [17].

The main aim of our study was to evaluate the prognostic utility of vitamin D measured as serum 25-hydroxyvitamin $\mathrm{D}[25(\mathrm{OH}) \mathrm{D}]$ in a Norwegian coastal coronary chest-pain population exposed to temperate sun activity at low altitudes and with a high consumption of fish as reflected by the omega-3 index. As a secondary aim, we looked into the seasonal correlation between $25(\mathrm{OH}) \mathrm{D}$ and the omega-3 index in the subjects with troponin-T ( $\mathrm{TnT}$ ) positive values.

\section{Methods}

Participants in the present analysis belonged to the RACS study (Risk Markers in the Acute Coronary Syndrome) (ClinicalTrials.gov NCT00521976), a single-center prospective observational study designed to evaluate the prognostic utility of serum $25(\mathrm{OH}) \mathrm{D}$ (D represents $\mathrm{D}_{2}$ and $\mathrm{D}_{3}$ ) status in 871 patients with chest pain and suspected ACS consecutively admitted to the Stavanger University Hospital from November 2002 to October 2003. The details of the RACS study have been published previously [18]. The primary outcome in the present study was all-cause mortality at 2and 7-year follow-up and the secondary outcomes included cardiac death and sudden cardiac death (SCD) at 2-year follow-up, as previously defined [16]. Measurements of the omega-3 index at admission were previously investigated in 456 of the 471 ACS patients (defined by a peak baseline TnT value $>0.01 \mathrm{ng} / \mathrm{mL}$ ) [19]. In this subpopulation we performed a seasonal (summer-autumn defined as JuneNovember and winter-spring defined as December-May) correlation between levels of $25(\mathrm{OH}) \mathrm{D}$ and the omega-3 index. $25(\mathrm{OH}) \mathrm{D}$ as a prognostic marker was evaluated in the total population, as well as in subgroups including TnT positive and TnT negative patients, gender, and vitamin $\mathrm{D}$ deficient subjects (defined as $25(\mathrm{OH}) \mathrm{D}<40 \mathrm{nM}(16 \mathrm{ng} / \mathrm{mL})$ ) [20]. Furthermore, seasonal variations in $25(\mathrm{OH}) \mathrm{D}$ were evaluated in the total population. Data were analyzed in quartiles of $25(\mathrm{OH}) \mathrm{D}$ and as continuous $25(\mathrm{OH}) \mathrm{D}$ values applying univariate and multivariable analyses.

The study was approved by the Regional Board of Research Ethics and the Norwegian Health Authorities and conducted in accordance with the Helsinki Declaration of 1971, as revised in 1983. Written informed consent was obtained from all patients.
Blood samples were drawn immediately following admission by direct venipuncture of an antecubital vein, applying a minimum of stasis. A second blood sample for TnT determination was drawn six hours following the first sample. Baseline laboratory data for this study included measurements of $25(\mathrm{OH}) \mathrm{D}_{2}, 25(\mathrm{OH}) \mathrm{D}_{3}$, TnT, high sensitivity Creactive protein (hsCRP), glucose, serum lipids, B-type natriuretic peptide (BNP) measured in EDTA (ethylene diamine tetraacetic acid) plasma, estimated glomerular filtration rate (eGFR) (calculated by modification in diet in renal disease (MDRD) formula), and omega-3 index.

TnT was quantified by a cardiac-specific second-generation troponin T ELISA assay from Roche Diagnostics, using a high-affinity cardiac-specific TnT isoform antibody [21]. The lower detection limit of the assay used is $0.01 \mathrm{ng} / \mathrm{mL}$.

$25(\mathrm{OH}) \mathrm{D}$ analysis was carried out at the Department of Medical Biochemistry at Stavanger University Hospital, as previously described [16].

The omega-3 index (EPA + DEA) content was measured in packed red blood cells, as previously described by Harris and von Schacky [9] and by Aarsetoey et al. [19].

2.1. Statistical Analysis. Patients were grouped into quartiles according to their $25(\mathrm{OH}) \mathrm{D}$ levels within the total population and TnT positive and negative subpopulations as appropriate. As a sensitivity analysis, selected analyses were conducted using continuous values instead of quartiles. Approximately normally distributed variables were given as mean and standard deviation (SD). The Chi-square test for association was applied between the $25(\mathrm{OH}) \mathrm{D}$ quartiles and categorical variables at baseline. The one way analysis of variance (ANOVA) was used to test for equality of means of scale variables (e.g., age) amongst $25(\mathrm{OH}) \mathrm{D}$ quartiles. The hazard ratios (HR) are presented with $95 \%$ confidence interval (CI). Separate stepwise Cox multivariable proportional hazards regression models with total death, cardiac death and SCD as the dependent variable, and $25(\mathrm{OH}) \mathrm{D}$ and other variables as potential independent predictors (listed below) were fitted. To examine the differences in prognosis between subjects in the upper quartile(s) versus the lowest quartile of $25(\mathrm{OH}) \mathrm{D}$, we adjusted for gender, age, smoking, hypertension, index diagnosis, diabetes mellitus, congestive heart failure (CHF) (defined by Killip-Kimball class at admission; patients in class 2 to 4 were classified as $\mathrm{CHF}$ patients and those in class 1 as non-CHF), history of previous coronary heart disease (CHD) (i.e., history of either angina pectoris, myocardial infarction, percutaneous coronary intervention, or coronary artery bypass graft), hypercholesterolemia/use of statins, TnT $>0.01 \mathrm{ng} / \mathrm{mL}$, eGFR, hsCRP, BNP, body mass index $\left(\mathrm{kg} / \mathrm{m}^{2}\right)$, and beta-blockers prior to enrolment. The Kaplan-Meier product limits were used for plotting times to event with the equality of the $25(\mathrm{OH}) \mathrm{D}$ quartile survival curves assessed by the log-rank test. Hypothesis two-sided $P$ values less than 0.05 are defined as statistically significant.

\section{Results}

A total of 871 patients (531 men and 340 women) were enrolled in the RACS study. Ten samples were not available; 
TABLE 1: Baseline characteristics of the total population arranged according to the quartiles of 25(OH)D.

\begin{tabular}{|c|c|c|c|c|c|}
\hline \multirow{2}{*}{ Characteristics $n(\%)$} & \multicolumn{4}{|c|}{ Quartiles of 25(OH)D } & \multirow{2}{*}{$P$ value } \\
\hline & Q1 & Q2 & Q3 & Q4 & \\
\hline $25(\mathrm{OH}) \mathrm{D}(\mathrm{nmol} / \mathrm{L})^{*}$ & $30.5 \pm 5.9$ & $45.2 \pm 3.4$ & $56.7 \pm 3.6$ & $78.6 \pm 13.2$ & $<0.001$ \\
\hline $25(\mathrm{OH}) \mathrm{D}(\mathrm{ng} / \mathrm{mL})^{*}$ & $12.2 \pm 2.4$ & $18.1 \pm 1.4$ & $22.7 \pm 1.4$ & $31.4 \pm 5.3$ & $<0.001$ \\
\hline Age, years* & $68.9 \pm 16.1$ & $69.4 \pm 15.6$ & $70.7 \pm 13.2$ & $69.3 \pm 12.4$ & 0.591 \\
\hline Male, $n(\%)$ & $120(55.8)$ & $138(64.2)$ & $127(58.8)$ & $140(65.1)$ & 0.150 \\
\hline Smoking status, $n(\%)$ & & & & & 0.110 \\
\hline Current smoker, $n(\%)$ & $71(33.0)$ & $58(27.0)$ & $46(21.3)$ & $52(24.2)$ & \\
\hline Past smoker, $n(\%)$ & $65(30.2)$ & $78(36.3)$ & $83(38.4)$ & $87(40.5)$ & \\
\hline Never smoked, $n(\%)$ & $79(36.7)$ & $79(36.7)$ & $87(40.3)$ & $76(35.3)$ & \\
\hline Angina pectoris, $n(\%)$ & $93(43.3)$ & $90(41.9)$ & $100(46.3)$ & $93(43.3)$ & 0.820 \\
\hline \multicolumn{6}{|l|}{$\mathrm{CHF}, n(\%)$} \\
\hline Killip Class 2-4 & $84(39.1)$ & $48(22.3)$ & $52(24.1)$ & $48(22.3)$ & $<0.001$ \\
\hline History of previous MI, $n(\%)$ & $82(38.1)$ & $69(32.1)$ & $71(32.9)$ & $66(30.7)$ & 0.382 \\
\hline $\mathrm{CABG}, n(\%)$ & $22(10.2)$ & $17(7.9)$ & $26(12.0)$ & $22(10.2)$ & 0.564 \\
\hline PCI, $n(\%)$ & $18(8.4)$ & $21(9.8)$ & 25 (11.6) & $23(10.7)$ & 0.721 \\
\hline Hypertension, $n(\%)$ & $87(40.5)$ & $86(40.0)$ & $96(44.4)$ & $93(43.3)$ & 0.746 \\
\hline History of DM $1, n(\%)$ & $2(0.9)$ & $2(0.9)$ & $0(0.0)$ & $4(1.9)$ & 0.256 \\
\hline History of DM $2, n(\%)$ & 38 (17.7) & $24(11.2)$ & $34(15.7)$ & $15(7.0)$ & 0.004 \\
\hline STEMI, $n(\%)$ & $30(14.0)$ & $35(16.3)$ & $31(14.4)$ & $32(14.9)$ & 0.913 \\
\hline TnT release, $n(\%)$ & $117(54.4)$ & $115(53.5)$ & $120(55.6)$ & $115(53.5)$ & 0.969 \\
\hline $\left.\operatorname{eGFR}(\mu \mathrm{mol} \mathrm{L})^{-1}\right)^{*}$ & $63.5 \pm 22.1$ & $61.7 \pm 20.8$ & $62.9 \pm 20.2$ & $63.0 \pm 20.3$ & 0.834 \\
\hline Cholesterol/Statin, $n(\%)$ & $104(48.4)$ & $105(48.8)$ & $109(50.5)$ & $107(49.8)$ & 0.973 \\
\hline Beta-blocker, $n(\%)$ & $81(37.7)$ & $78(36.3)$ & $77(35.6)$ & $73(34.0)$ & 0.881 \\
\hline Known CHD, $n(\%)$ & $140(65.1)$ & $132(61.4)$ & $142(65.7)$ & $129(60.0)$ & 0.537 \\
\hline $\operatorname{BMI}\left(\mathrm{kg} / \mathrm{m}^{2}\right)^{*}$ & $25.7 \pm 4.4$ & $26.3 \pm 4.7$ & $25.7 \pm 4.2$ & $25.0 \pm 3.7$ & 0.024 \\
\hline BNP quartiles & & & & & 0.222 \\
\hline Q1 & $47(22.8)$ & $52(24.8)$ & $44(21.4)$ & $64(31.7)$ & \\
\hline Q2 & $50(24.3)$ & $50(23.8)$ & $60(29.1)$ & $44(21.8)$ & \\
\hline Q3 & $48(23.3)$ & $53(25.2)$ & $54(26.2)$ & $53(26.2)$ & \\
\hline Q4 & $61(29.6)$ & $55(26.2)$ & $48(23.3)$ & $41(20.3)$ & \\
\hline hsCRP quartiles & & & & & 0.910 \\
\hline Q1 & $51(23.7)$ & $52(24.2)$ & $53(24.5)$ & $61(28.4)$ & \\
\hline Q2 & $49(22.8)$ & $50(23.3)$ & $56(25.9)$ & $57(26.5)$ & \\
\hline Q3 & $57(26.5)$ & $57(26.5)$ & $53(24.5)$ & $50(23.3)$ & \\
\hline Q4 & $58(27.0)$ & $56(26.0)$ & $54(25.0)$ & 47 (21.9) & \\
\hline
\end{tabular}

* Mean \pm SD.

SD, standard deviation; 25(OH)D, 25-hydroxyvitamin D; CHF, congestive heart failure; MI, myocardial infarction; CABG, coronary artery bypass grafting; PCI, percutaneous coronary intervention; DM, diabetes mellitus; STEMI, ST-elevation myocardial infarction; TnT, troponin T; eGFR, estimated glomerular filtration rate; CHD, coronary heart disease; BMI, body mass index; BNP, B-type natriuretic peptide; hsCRP, high sensitivity C-reactive protein.

thus 861 patients (525 men and 336 women) were included in the present $25(\mathrm{OH}) \mathrm{D}$ analysis. Of these, 467 patients $(54 \%)$ (305 men and 162 women) were characterized by a TnT release $>0.01 \mathrm{ng} / \mathrm{mL}$. Mean age in the total patient population was $69.6 \pm 14.4$ years (males $66.6 \pm 14.3$ years and females $74.4 \pm 13.1$ years). Mean 25(OH)D levels were $52.7 \pm 19.1 \mathrm{nM}$ $(21.1 \pm 7.6 \mathrm{ng} / \mathrm{mL})$ in the total patient population; males $53.8 \pm$ $19.3 \mathrm{nM}(21.5 \pm 7.7 \mathrm{ng} / \mathrm{mL})$ and females $51.0 \pm 18.8 \mathrm{nM}(20.4 \pm$ $7.5 \mathrm{ng} / \mathrm{mL})$. Seasonal $25(\mathrm{OH}) \mathrm{D}$ levels in all patients were $51.6 \pm 18.9 \mathrm{nM}(20.6 \pm 7.6 \mathrm{ng} / \mathrm{mL})$ during winter-spring and $55.4 \pm 19.1 \mathrm{nM}(22.2 \pm 7.6 \mathrm{ng} / \mathrm{mL})$ during summer-autumn; $P=0.007$. The seasonal difference was greatest in males
$(52.1 \mathrm{nM} \pm 19.1 \mathrm{nM}$ versus $57.7 \mathrm{nM} \pm 19.1 \mathrm{nM} ; P=0.002)$ and not significant in women $(50.7 \mathrm{nM} \pm 18.5 \mathrm{nM}$ versus $51.6 \mathrm{nM}$ $\pm 19.5 \mathrm{nM} ; P=0.711$ ).

The baseline characteristics according to $25(\mathrm{OH}) \mathrm{D}$ quartiles at admission in the total patient population, in the TnT positive group, and in both genders are shown in Tables 1, 2, and 3(a)-3(b), respectively.

3.1. 2-Year Follow-Up. At 2-year follow-up, 136 (70 men and 66 women) patients with known 25(OH)D values had died, $84(62 \%)$ of whom died due to a cardiac event, and of these, 25 cases were defined as SCD. In the TnT positive group 104 
TABLE 2: Baseline characteristics of the TnT positive patient population arranged according to the quartiles of 25(OH)D.

\begin{tabular}{|c|c|c|c|c|c|}
\hline \multirow{2}{*}{ Characteristics $n(\%)$} & \multicolumn{4}{|c|}{ Quartiles of 25(OH)D } & \multirow{2}{*}{$P$ value } \\
\hline & Q1 & Q2 & Q3 & Q4 & \\
\hline $25(\mathrm{OH}) \mathrm{D}(\mathrm{nmol} / \mathrm{L})^{*}$ & $30.2 \pm 6.2$ & $44.9 \pm 3.5$ & $56.6 \pm 3.6$ & $78.1 \pm 13.2$ & $<0.001$ \\
\hline $25(\mathrm{OH}) \mathrm{D}(\mathrm{ng} / \mathrm{mL})^{*}$ & $12.1 \pm 2.5$ & $18.0 \pm 1.4$ & $22.6 \pm 1.4$ & $31.2 \pm 5.3$ & $<0.001$ \\
\hline Omega-3 Index ${ }^{*}$ & $6.1 \pm 1.6$ & $6.4 \pm 1.6$ & $6.9 \pm 1.9$ & $7.5 \pm 2.2$ & $<0.001$ \\
\hline Age, years* & $72.5 \pm 14.8$ & $72.6 \pm 13.5$ & $71.5 \pm 13.8$ & $71.2 \pm 11.0$ & 0.809 \\
\hline Male, $n(\%)$ & $64(55.2)$ & $81(69.2)$ & $73(62.4)$ & $86(74.1)$ & 0.015 \\
\hline Smoking status, $n(\%)$ & & & & & 0.047 \\
\hline Current smoker, $n(\%)$ & $36(31.0)$ & $37(31.6)$ & $35(29.9)$ & $30(25.9)$ & \\
\hline Past smoker, $n(\%)$ & $30(25.9)$ & $42(35.9)$ & $35(29.9)$ & $53(45.7)$ & \\
\hline Never smoked, $n(\%)$ & $50(43.1)$ & $38(32.5)$ & $47(40.2)$ & $33(28.4)$ & \\
\hline Angina pectoris, $n(\%)$ & $49(42.2)$ & $48(41.0)$ & $52(44.4)$ & $51(44.0)$ & 0.949 \\
\hline \multicolumn{6}{|l|}{ CHF, $n(\%)$} \\
\hline Killip Class 2-4 & $56(48.3)$ & $27(23.1)$ & $30(25.6)$ & $32(27.6)$ & $<0.001$ \\
\hline History of previous MI, $n(\%)$ & $46(39.7)$ & $37(31.6)$ & $35(29.9)$ & $38(32.8)$ & 0.413 \\
\hline $\mathrm{CABG}, n(\%)$ & $11(9.5)$ & $7(6.0)$ & $12(10.3)$ & $12(10.3)$ & 0.612 \\
\hline PCI, $n(\%)$ & $9(7.8)$ & $9(7.7)$ & $7(6.0)$ & $10(8.6)$ & 0.893 \\
\hline Hypertension, $n(\%)$ & $48(41.4)$ & $47(40.2)$ & $52(44.4)$ & $49(42.2)$ & 0.926 \\
\hline History of DM $1, n(\%)$ & $0(0.0)$ & $2(1.7)$ & $0(0.0)$ & $4(3.4)$ & 0.058 \\
\hline History of DM 2, $n(\%)$ & $26(22.4)$ & $19(16.2)$ & $19(16.2)$ & $9(7.8)$ & 0.023 \\
\hline STEMI, $n(\%)$ & $29(25.0)$ & $35(29.9)$ & $30(25.6)$ & $33(28.4)$ & 0.813 \\
\hline $\left.\mathrm{eGFR}(\mu \mathrm{mol} \mathrm{L})^{-1}\right)^{*}$ & $59.7 \pm 23.2$ & $59.8 \pm 22.3$ & $62.5 \pm 23.3$ & $63.9 \pm 23.5$ & 0.418 \\
\hline Cholesterol/statin, $n(\%)$ & $53(45.7)$ & $54(46.2)$ & $52(44.4)$ & $50(43.1)$ & 0.967 \\
\hline Beta-blocker, $n(\%)$ & $40(34.5)$ & $39(33.3)$ & $35(29.9)$ & $34(29.3)$ & 0.793 \\
\hline Known CHD, $n(\%)$ & $80(69.0)$ & $71(60.7)$ & $76(65.0)$ & $72(62.1)$ & 0.566 \\
\hline $\operatorname{BMI}\left(\mathrm{kg} / \mathrm{m}^{2}\right)^{*}$ & $25.7 \pm 4.5$ & $25.9 \pm 4.6$ & $25.6 \pm 4.3$ & $25.3 \pm 3.9$ & 0.714 \\
\hline BNP quartiles & & & & & 0.778 \\
\hline Q1 & $15(13.8)$ & $17(15.0)$ & $22(19.5)$ & $23(21.3)$ & \\
\hline Q2 & $24(22.0)$ & $23(20.4)$ & $25(22.1)$ & $24(22.2)$ & \\
\hline Q3 & $26(23.9)$ & $34(30.1)$ & $32(28.3)$ & $26(24.1)$ & \\
\hline Q4 & $44(40.4)$ & $39(34.5)$ & $34(30.1)$ & $35(32.4)$ & \\
\hline hsCRP quartiles & & & & & 0.698 \\
\hline Q1 & $18(15.5)$ & $22(18.8)$ & $19(16.2)$ & $27(23.3)$ & \\
\hline Q2 & $24(20.7)$ & $27(23.1)$ & $31(26.5)$ & $27(23.3)$ & \\
\hline Q3 & $32(27.6)$ & $36(30.8)$ & $36(30.8)$ & $32(27.6)$ & \\
\hline Q4 & $42(36.2)$ & $32(27.4)$ & $31(26.5)$ & $30(25.9)$ & \\
\hline
\end{tabular}

${ }^{*}$ Mean \pm SD.

SD, standard deviation; 25(OH)D, 25-hydroxyvitamin D; CHF, congestive heart failure; MI, myocardial infarction; CABG, coronary artery bypass grafting; PCI, percutaneous coronary intervention; DM, diabetes mellitus; STEMI, ST-elevation myocardial infarction; TnT, troponin T; eGFR, estimated glomerular filtration rate; $\mathrm{CHD}$, coronary heart disease; $\mathrm{BMI}$, body mass index; $\mathrm{BNP}, \mathrm{B}$-type natriuretic peptide; hsCRP, high sensitivity C-reactive protein.

patients with known 25(OH)D values died: $71(68 \%)$ due to a cardiac event, including 21 sudden cardiac deaths.

In the univariate quartile analysis of all-cause mortality in the total population and in the TnT positive patient population, the HRs for $25(\mathrm{OH}) \mathrm{D}$ in the highest quartile (Q4) as compared to the lowest quartile (Q1) were 0.61 (95\% CI, 0.37-1.00), $P=0.050$, and 0.59 (95\% CI, 0.33-1.04), $P=$ 0.067 , respectively. No difference in $\mathrm{HR}$ was found between $25(\mathrm{OH}) \mathrm{D}$ quartiles in the TnT negative population, nor for cardiac death in the total and TnT positive population. The results remained statistically nonsignificant in the multivariable analysis for all groups. The quartile univariate and multivariable results are presented in Table 4.

In the univariate analysis of continuous $25(\mathrm{OH}) \mathrm{D}$ values in the total population, the HRs for total mortality and cardiac death were 0.99 (95\% CI, 0.98-1.00), $P=0.035$, and 0.99 (95\% CI, $0.98-1.00), P=0.060$, respectively. In the univariate analysis of the TnT positive population, the HRs 
TABLE 3: (a) Baseline characteristics of the female population arranged according to the quartiles of 25(OH)D. (b) Baseline characteristics of the male population arranged according to the quartiles of $25(\mathrm{OH}) \mathrm{D}$.

(a)

\begin{tabular}{|c|c|c|c|c|c|}
\hline \multirow{2}{*}{ Characteristics $n(\%)$} & \multicolumn{4}{|c|}{ Quartiles of 25(OH)D } & \multirow{2}{*}{$P$ value } \\
\hline & Q1 & Q2 & Q3 & Q4 & \\
\hline $25(\mathrm{OH}) \mathrm{D}(\mathrm{nmol} / \mathrm{L})^{*}$ & $28.8 \pm 5.6$ & $43.2 \pm 3.9$ & $55.6 \pm 3.4$ & $76.1 \pm 12.3$ & $<0.001$ \\
\hline $25(\mathrm{OH}) \mathrm{D}(\mathrm{ng} / \mathrm{mL})^{*}$ & $11.5 \pm 2.2$ & $17.3 \pm 1.6$ & $22.2 \pm 1.4$ & $30.4 \pm 4.9$ & $<0.001$ \\
\hline Age, years* & $75.5 \pm 14.3$ & $75.8 \pm 12.9$ & $75.0 \pm 12.1$ & $70.9 \pm 12.8$ & 0.058 \\
\hline Smoking status, $n(\%)$ & & & & & 0.384 \\
\hline Current smoker, $n(\%)$ & $18(21.7)$ & $14(16.7)$ & $10(11.9)$ & $17(20.2)$ & \\
\hline Past smoker, $n(\%)$ & $13(15.7)$ & $21(25.0)$ & $17(20.2)$ & $21(25.0)$ & \\
\hline Never smoked, $n(\%)$ & $52(62.7)$ & $49(58.3)$ & $57(67.9)$ & $46(54.8)$ & \\
\hline Angina pectoris, $n(\%)$ & $44(53.0)$ & $45(53.6)$ & $39(46.3)$ & $39(46.4)$ & 0.663 \\
\hline \multicolumn{6}{|l|}{$\mathrm{CHF}, n(\%)$} \\
\hline Killip class $2-4$ & $43(51.8)$ & $26(31.0)$ & $20(23.8)$ & $17(20.2)$ & $<0.001$ \\
\hline History of previous MI, $n(\%)$ & $30(36.1)$ & $25(29.8)$ & $25(29.8)$ & $22(26.2)$ & 0.567 \\
\hline $\mathrm{CABG}, n(\%)$ & $6(7.2)$ & $3(3.6)$ & $8(9.5)$ & $3(3.6)$ & 0.279 \\
\hline PCI, $n(\%)$ & $1(1.2)$ & $2(2.4)$ & $7(8.3)$ & $8(9.5)$ & 0.035 \\
\hline Hypertension, $n(\%)$ & $41(49.4)$ & $39(46.4)$ & $40(47.6)$ & $37(44.4)$ & 0.917 \\
\hline History of DM $1, n(\%)$ & $1(1.2)$ & $1(1.2)$ & $0(0.0)$ & $1(1.2)$ & 0.798 \\
\hline History of DM $2, n(\%)$ & $11(13.3)$ & $12(14.3)$ & $13(15.5)$ & $9(10.7)$ & 0.828 \\
\hline STEMI, $n(\%)$ & $11(13.3)$ & $7(8.3)$ & $3(3.6)$ & $7(8.3)$ & 0.164 \\
\hline TnT release, $n(\%)$ & $46(55.4)$ & $40(47.6)$ & $42(50.0)$ & $34(40.5)$ & 0.277 \\
\hline eGFR $\left(\mu \mathrm{mol} \mathrm{L}^{-1}\right)^{*}$ & $52.3 \pm 18.4$ & $54.9 \pm 17.4$ & $53.9 \pm 17.8$ & $58.9 \pm 15.1$ & 0.079 \\
\hline Cholesterol/statin, $n(\%)$ & $43(51.8)$ & $46(54.8)$ & $45(53.6)$ & $45(53.6)$ & 0.985 \\
\hline Beta-blocker, $n(\%)$ & $33(39.8)$ & $32(38.1)$ & $38(45.2)$ & $23(27.4)$ & 0.112 \\
\hline Known CHD, $n(\%)$ & $59(71.1)$ & $56(66.7)$ & $56(66.7)$ & $50(59.5)$ & 0.465 \\
\hline BMI $\left(\mathrm{kg} / \mathrm{m}^{2}\right)^{*}$ & $25.4 \pm 4.8$ & $25.5 \pm 4.6$ & $25.2 \pm 5.2$ & $24.9 \pm 4.0$ & 0.886 \\
\hline BNP quartiles & & & & & 0.094 \\
\hline Q1 & $17(20.7)$ & $20(25.0)$ & $17(21.5)$ & $27(33.8)$ & \\
\hline Q2 & $19(23.2)$ & $23(28.8)$ & $20(25.3)$ & $19(23.8)$ & \\
\hline Q3 & $15(18.3)$ & $21(26.3)$ & $23(29.1)$ & $21(26.3)$ & \\
\hline Q4 & $31(37.8)$ & $16(20.0)$ & $19(24.1)$ & $13(16.3)$ & \\
\hline hsCRP quartiles & & & & & 0.995 \\
\hline Q1 & $20(24.1)$ & $19(22.6)$ & $21(25.0)$ & $24(28.6)$ & \\
\hline Q2 & $21(25.3)$ & $20(23.8)$ & $22(26.2)$ & $20(23.8)$ & \\
\hline Q3 & $21(25.3)$ & $22(26.2)$ & $19(22.6)$ & $22(26.2)$ & \\
\hline Q4 & $21(25.3)$ & $23(27.4)$ & $22(26.2)$ & $18(21.4)$ & \\
\hline
\end{tabular}

${ }^{*}$ Mean \pm SD.

SD, standard deviation; 25(OH)D, 25-hydroxyvitamin D; CHF, congestive heart failure; MI, myocardial infarction; CABG, coronary artery bypass grafting; PCI, percutaneous coronary intervention; DM, diabetes mellitus; STEMI, ST-elevation myocardial infarction; TnT, troponin T; eGFR, estimated glomerular filtration rate; $\mathrm{CHD}$, coronary heart disease; BMI, body mass index; BNP, B-type natriuretic peptide; hsCRP, high sensitivity C-reactive protein.

(b)

\begin{tabular}{|c|c|c|c|c|c|}
\hline \multirow{2}{*}{ Characteristics $n(\%)$} & \multicolumn{4}{|c|}{ Quartiles of 25(OH)D } & \multirow{2}{*}{$P$ value } \\
\hline & Q1 & Q2 & Q3 & Q4 & \\
\hline $25(\mathrm{OH}) \mathrm{D}(\mathrm{nmol} / \mathrm{L})^{*}$ & $31.7 \pm 6.0$ & $46.3 \pm 3.0$ & $57.4 \pm 3.8$ & $79.9 \pm 13.7$ & $<0.001$ \\
\hline $25(\mathrm{OH}) \mathrm{D}(\mathrm{ng} / \mathrm{mL})^{*}$ & $12.7 \pm 2.4$ & $18.5 \pm 1.2$ & $23.0 \pm 1.5$ & $32.0 \pm 5.5$ & $<0.001$ \\
\hline Age, years* & $63.6 \pm 15.3$ & $66.0 \pm 16.3$ & $67.8 \pm 12.9$ & $68.6 \pm 12.3$ & 0.024 \\
\hline Smoking status, $n(\%)$ & & & & & 0.211 \\
\hline Current smoker, $n(\%)$ & $53(40.5)$ & $44(33.6)$ & $37(28.0)$ & $34(26.0)$ & \\
\hline Past smoker, $n(\%)$ & $53(40.5)$ & $58(44.3)$ & $67(50.8)$ & $63(48.1)$ & \\
\hline Never smoked, $n(\%)$ & $25(19.1)$ & $29(22.1)$ & $28(21.2)$ & $34(26.0)$ & \\
\hline
\end{tabular}


(b) Continued.

\begin{tabular}{|c|c|c|c|c|c|}
\hline \multirow{2}{*}{ Characteristics $n(\%)$} & \multicolumn{4}{|c|}{ Quartiles of 25(OH)D } & \multirow{2}{*}{$P$ value } \\
\hline & Q1 & Q2 & Q3 & Q4 & \\
\hline Angina pectoris, $n(\%)$ & $46(35.1)$ & $48(36.6)$ & $60(45.5)$ & $54(41.2)$ & 0.306 \\
\hline \multicolumn{6}{|l|}{ CHF, $n(\%)$} \\
\hline Killip Class 2-4 & $42(32.1)$ & $21(16.0)$ & $32(24.2)$ & $31(23.7)$ & 0.026 \\
\hline History of previous MI, $n(\%)$ & $50(38.2)$ & $46(35.1)$ & $45(34.1)$ & $44(33.6)$ & 0.867 \\
\hline $\mathrm{CABG}, n(\%)$ & $18(13.7)$ & $14(10.7)$ & $16(12.1)$ & $19(14.5)$ & 0.795 \\
\hline PCI, $n(\%)$ & $19(14.5)$ & $17(13.0)$ & $18(13.6)$ & $15(11.5)$ & 0.903 \\
\hline Hypertension, $n(\%)$ & $44(33.6)$ & $45(34.4)$ & $56(42.4)$ & $59(45.0)$ & 0.141 \\
\hline History of DM $1, n(\%)$ & $1(0.8)$ & $1(0.8)$ & $1(0.8)$ & $2(1.5)$ & 0.894 \\
\hline History of DM $2, n(\%)$ & $25(19.1)$ & $14(10.7)$ & $19(14.4)$ & $8(6.1)$ & 0.013 \\
\hline STEMI, $n(\%)$ & $21(16.0)$ & $28(21.4)$ & $28(21.2)$ & $23(17.6)$ & 0.610 \\
\hline TnT release, $n(\%)$ & $73(55.7)$ & $74(56.5)$ & $76(57.6)$ & $81(61.8)$ & 0.754 \\
\hline eGFR $\left(\mu \mathrm{mol} \mathrm{L}^{-1}\right)^{*}$ & $70.5 \pm 21.7$ & $67.0 \pm 21.0$ & $68.1 \pm 20.6$ & $65.8 \pm 21.7$ & 0.312 \\
\hline Cholesterol/statin, $n(\%)$ & $57(43.5)$ & $62(47.3)$ & $65(49.2)$ & $61(46.6)$ & 0.826 \\
\hline Beta-blocker, $n(\%)$ & $49(37.4)$ & $44(33.6)$ & $44(33.3)$ & $45(34.4)$ & 0.894 \\
\hline Known CHD, $n(\%)$ & $80(61.1)$ & $77(58.8)$ & $85(64.4)$ & $79(60.3)$ & 0.817 \\
\hline $\operatorname{BMI}\left(\mathrm{kg} / \mathrm{m}^{2}\right)^{*}$ & $26.1 \pm 3.9$ & $26.6 \pm 4.9$ & $26.0 \pm 3.4$ & $25.0 \pm 3.5$ & 0.014 \\
\hline BNP quartiles & & & & & 0.545 \\
\hline Q1 & $29(23.4)$ & $35(27.3)$ & $27(21.1)$ & $36(29.5)$ & \\
\hline Q2 & $30(24.2)$ & $30(23.4)$ & $39(30.5)$ & $27(22.1)$ & \\
\hline Q3 & $30(24.2)$ & $27(21.1)$ & $36(28.1)$ & $31(25.4)$ & \\
\hline Q4 & $35(28.2)$ & $36(28.1)$ & $26(20.3)$ & $28(23.0)$ & \\
\hline hsCRP Quartiles & & & & & 0.904 \\
\hline Q1 & $30(22.9)$ & $33(25.2)$ & $32(24.2)$ & $36(27.5)$ & \\
\hline Q2 & $28(21.4)$ & $31(23.7)$ & $35(26.5)$ & $36(27.5)$ & \\
\hline Q3 & $37(28.2)$ & $34(26.0)$ & $35(26.5)$ & $27(20.6)$ & \\
\hline Q4 & $36(27.5)$ & $33(25.2)$ & $30(22.7)$ & $32(24.4)$ & \\
\hline
\end{tabular}

* Mean \pm SD.

SD, standard deviation; 25(OH)D, 25-hydroxyvitamin D; CHF, congestive heart failure; MI, myocardial infarction; CABG, coronary artery bypass grafting; PCI, percutaneous coronary intervention; DM, diabetes mellitus; STEMI, ST-elevation myocardial infarction; TnT, troponin T; eGFR, estimated glomerular filtration rate; $\mathrm{CHD}$, coronary heart disease; $\mathrm{BMI}$, body mass index; $\mathrm{BNP}$, B-type natriuretic peptide; hsCRP, high sensitivity C-reactive protein.

for total mortality and cardiac death were 0.99 (95\% CI, 0.99-1.00), $P=0.012$, and 0.99 (95\% CI, 0.98-1.00), $P=$ 0.055 , respectively. The remaining continuous univariate and multivariable results are shown in Table 5.

3.2. 2-Year Gender Analysis. In the univariate quartile analysis of total and cardiac mortality in the female population, the HRs for 25(OH)D in Q4 as compared to Q1 were 0.43 (95\% CI, 0.19-0.94), $P=0.035$, and 0.35 (95\% CI, 0.130.98), $P=0.046$, respectively. No difference in the $\mathrm{HR}$ was found between the $25(\mathrm{OH}) \mathrm{D}$ quartiles in males. The $25(\mathrm{OH}) \mathrm{D}$ quartiles' HRs for SCD were not significant in either the univariate or multivariable models. The quartile univariate and multivariable results are presented in Table 4.

In the univariate analysis of continuous $25(\mathrm{OH}) \mathrm{D}$ values in the female population, the HRs for total mortality and cardiac death were 0.98 (95\% CI, 0.97-1.00), $P=0.023$, and 0.98 (95\% CI, 0.97-1.00), $P=0.044$, respectively. The continuous univariate and multivariable results are presented in Table 5.
3.3. 7-Year Follow-Up. At 7-year follow-up, data collection was restricted to total mortality. During this period 327 (180 men and 147 women) patients had died. The Kaplan-Meier curve for survival is shown in Figure 1. In the TnT positive group, 216 patients (46\%) died, as shown in Figure 2.

In the univariate quartile analysis of all-cause mortality in the total population and in the TnT positive patient population, the HRs for 25(OH)D in Q4 as compared to Q1 were 0.66 (95\% CI, 0.48-0.89), $P=0.007$, and 0.60 (95\% CI, 0.41$0.87), P=0.008$, respectively. No difference in mortality was found amongst the $25(\mathrm{OH}) \mathrm{D}$ quartiles in the TnT negative population. The results were statistically nonsignificant in the multivariable analysis for all groups. The quartile univariate and multivariable results are presented in Table 4.

In the univariate analysis of continuous $25(\mathrm{OH}) \mathrm{D}$ values in the total population and in the TnT positive patient population, the HRs for total mortality were 0.99 (95\% CI, 0.99-1.00), $P=0.026$, and 0.99 (95\% CI, 0.98-1.00), $P=0.002$, respectively. The continuous univariate and multivariable results are presented in Table 5. 
TABLE 4: The univariate and multivariable HRs (95\% CI) for quartiles of 25(OH)D.

\begin{tabular}{|c|c|c|c|c|}
\hline & \multicolumn{2}{|c|}{ 2-year follow-up } & \multicolumn{2}{|c|}{ 7-year follow-up } \\
\hline & Univariate analysis & Multivariable analysis* & Univariate analysis & Multivariable analysis* \\
\hline \multicolumn{5}{|l|}{ Total population } \\
\hline Total mortality & $\begin{array}{c}0.61(0.37-1.00) \\
P=0.05\end{array}$ & $\begin{array}{c}0.79(0.45-1.40) \\
P=0.40\end{array}$ & $\begin{array}{c}0.66(0.48-0.89) \\
P=0.008\end{array}$ & $\begin{array}{c}0.79(0.57-1.09) \\
P=0.15\end{array}$ \\
\hline Cardiac death & $\begin{array}{c}0.70(0.37-1.34) \\
P=0.29\end{array}$ & $\begin{array}{c}0.83(0.40-1.72) \\
P=0.61\end{array}$ & NA & NA \\
\hline SCD & $\begin{array}{c}0.59(0.14-2.44) \\
P=0.46\end{array}$ & $\begin{array}{c}1.28(0.26-6.37) \\
P=0.76 \\
\end{array}$ & NA & NA \\
\hline \multicolumn{5}{|c|}{ TnT pos. population } \\
\hline Total mortality & $\begin{array}{c}0.59(0.33-1.04) \\
P=0.07\end{array}$ & $\begin{array}{c}0.82(0.42-1.61) \\
P=0.56\end{array}$ & $\begin{array}{c}0.60(0.41-0.87) \\
P=0.008\end{array}$ & $\begin{array}{c}0.74(0.49-1.12) \\
P=0.15\end{array}$ \\
\hline Cardiac death & $\begin{array}{c}0.71(0.36-1.41) \\
P=0.33\end{array}$ & $\begin{array}{c}1.09(0.49-2.42) \\
P=0.84\end{array}$ & NA & NA \\
\hline SCD & $\begin{array}{c}0.58(0.14-2.44) \\
P=0.46\end{array}$ & $\begin{array}{c}1.52(0.30-7.72) \\
P=0.61\end{array}$ & NA & NA \\
\hline \multicolumn{5}{|l|}{ Females } \\
\hline Total mortality & $\begin{array}{c}0.43(0.19-0.94) \\
P=0.035\end{array}$ & $\begin{array}{c}0.65(0.26-1.62) \\
P=0.36\end{array}$ & $\begin{array}{c}0.51(0.32-0.83) \\
P=0.006\end{array}$ & $\begin{array}{c}0.64(0.38-1.10) \\
P=0.09\end{array}$ \\
\hline Cardiac death & $\begin{array}{c}0.35(0.13-0.98) \\
P=0.046\end{array}$ & $\begin{array}{c}0.61(0.19-2.00) \\
P=0.41\end{array}$ & NA & NA \\
\hline SCD & $\begin{array}{c}0.0(0.00-0.00) \\
P=0.96\end{array}$ & NA & NA & NA \\
\hline \multicolumn{5}{|l|}{ Males } \\
\hline Total mortality & $\begin{array}{c}0.85(0.46-1.57) \\
P=0.61\end{array}$ & $\begin{array}{c}0.81(0.40-1.65) \\
P=0.56\end{array}$ & $\begin{array}{c}0.82(0.56-1.24) \\
P=0.36\end{array}$ & $\begin{array}{c}0.73(0.47-1.12) \\
P=0.15\end{array}$ \\
\hline Cardiac death & $\begin{array}{c}1.09(0.46-2.56) \\
P=0.85\end{array}$ & $\begin{array}{c}0.89(0.32-2.46) \\
P=0.83\end{array}$ & NA & NA \\
\hline SCD & $\begin{array}{c}0.99(0.20-4.88) \\
P=0.99\end{array}$ & $\begin{array}{c}1.92(0.31-12.1) \\
P=0.49\end{array}$ & NA & NA \\
\hline
\end{tabular}

SCD, sudden cardiac death.

${ }^{*}$ Adjusted for gender, age, smoking, hypertension, index diagnosis, diabetes mellitus, CHF (defined by Killip-Kimball class at admission; patients in classes 2 to 4 were classified as CHF patients and those in class 1 as non-CHF), history of previous CHD (i.e., history of either angina pectoris, myocardial infarction, percutaneous coronary intervention, or coronary artery bypass graft), hypercholesterolemia/use of statins, TnT $>0.01 \mathrm{ng} / \mathrm{mL}$, eGFR, hsCRP, BNP, body mass index $\left(\mathrm{kg} / \mathrm{m}^{2}\right)$, and beta-blockers prior to enrolment.

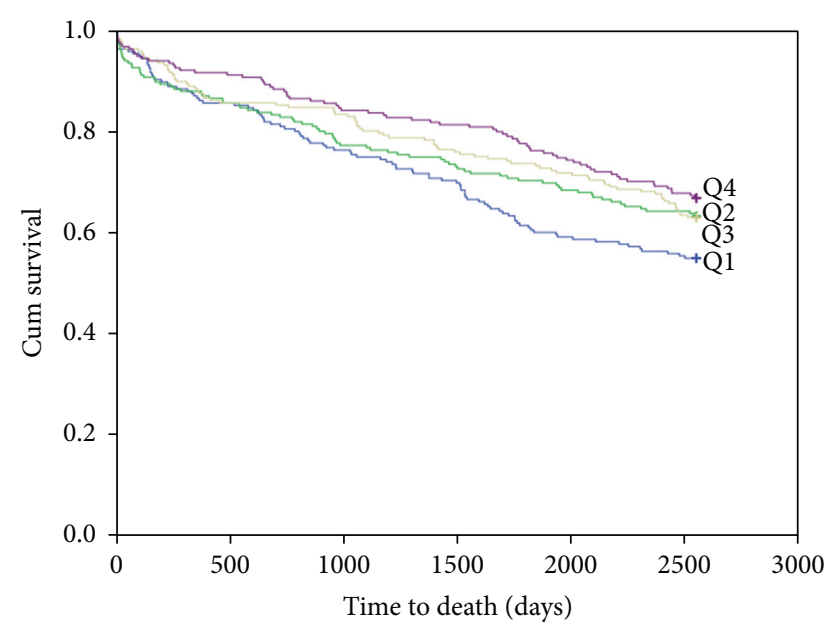

FIGURE 1: Kaplan-Meier plots for seven-year total mortality of $25(\mathrm{OH}) \mathrm{D}$ quartiles in the total patient population.

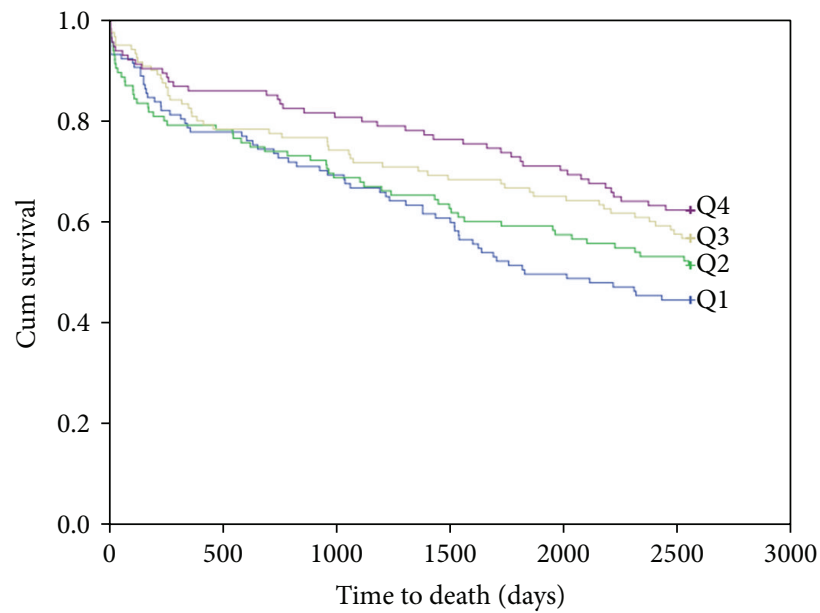

Figure 2: Kaplan-Meier plots for seven-year total mortality of 25(OH)D quartiles in the TnT positive patient population. 
TABLE 5: The univariate and multivariable HRs (95\% CI) for continuous 25(OH)D values.

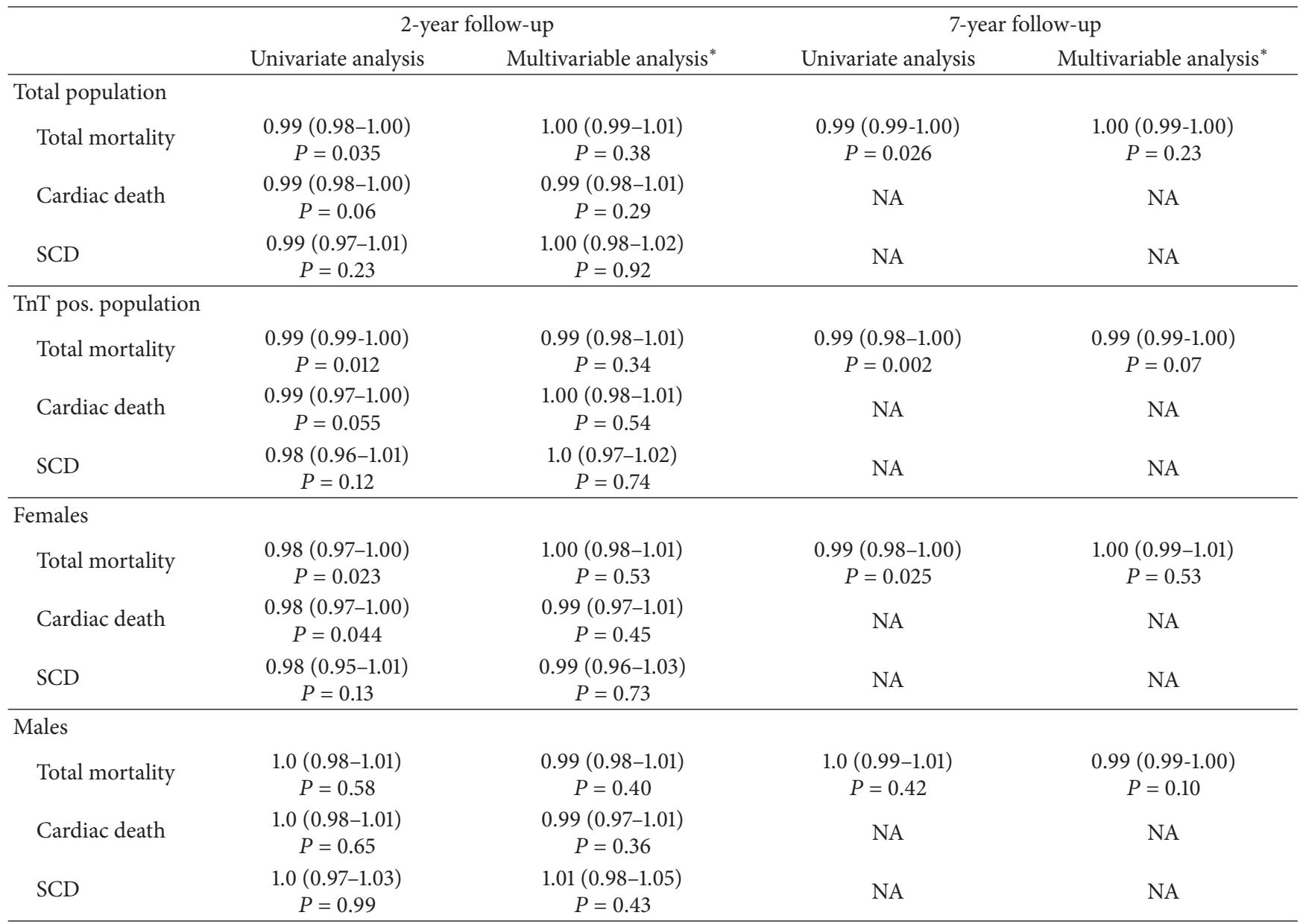

SCD, sudden cardiac death.

*Adjusted for gender, age, smoking, hypertension, index diagnosis, diabetes mellitus, CHF (defined by Killip-Kimball class at admission; patients in class 2 to 4 were classified as CHF patients and those in class 1 as non CHF), history of previous CHD (i.e., history of either angina pectoris, myocardial infarction, percutaneous coronary intervention, or coronary artery bypass graft), hypercholesterolemia/use of statins, TnT $>0.01 \mathrm{ng} / \mathrm{mL}$, eGFR, hsCRP, BNP, body mass index $\left(\mathrm{kg} / \mathrm{m}^{2}\right)$, and beta-blockers prior to enrolment.

Evaluating the influence of diabetes in the total population, the univariate analysis at 7 -year follow-up revealed a significant difference in total mortality, $P<0.001$, not found in the multivariable analysis.

3.4. 7-Year Gender Analysis. The univariate quartile analysis showed a statistically significant difference in the HR of Q4 as compared to Q1 of $25(\mathrm{OH}) \mathrm{D}$ for total death among females 0.51 (95\% CI, 0.32-0.83), $P=0.006$, but not amongst males. The KM curve for survival in females is shown in Figure 3. The difference found in women was no longer significant in the multivariable analysis. The quartile univariate and multivariable results are presented in Table 4.

In the univariate analysis of continuous $25(\mathrm{OH}) \mathrm{D}$ values in the female population, the HR for total mortality was 0.99 (95\% CI 0.98-1.00), $P=0.025$. The continuous univariate and multivariable results are presented in Table 5.

3.5. 7-Year Follow-Up of Vitamin D Deficient $[<40 \mathrm{nM}$ $(<16 \mathrm{ng} / \mathrm{mL})]$ Patients. There were 232 patients (27\%) with
$25(\mathrm{OH}) \mathrm{D}$ levels below $40 \mathrm{nM}(16 \mathrm{ng} / \mathrm{mL})$ and 629 patients (73\%) with $25(\mathrm{OH}) \mathrm{D}$ levels above and equal to $40 \mathrm{nM}$ $(16 \mathrm{ng} / \mathrm{mL})$. The univariate HR for total mortality in patients with 25(OH)D deficiency was 1.43 (95\% CI, 1.14-1.81); $P=$ 0.002 , as compared to subjects without $25(\mathrm{OH}) \mathrm{D}$ deficiency and was still significant in the multivariable model; HR 1.32 (95\% CI, 1.04-1.68), $P=0.024$.

3.6. Seasonal Correlation between Vitamin D and Omega-3 Index. 456 patients ( 298 men and 158 women) were included in the seasonal correlation study between $25(\mathrm{OH}) \mathrm{D}$ and omega-3 index. Mean 25(OH)D levels in this subgroup were $50.4 \pm 18.2 \mathrm{nM}(29.2 \pm 7.3 \mathrm{ng} / \mathrm{mL})$ winter-spring and $55.2 \pm$ $20.1 \mathrm{nM}(22.1 \pm 8.0 \mathrm{mg} / \mathrm{mL})$ during the summer-autumn season; $P=0.009$. Corresponding levels of the omega- 3 index were $6.8(1.9) \%$ and $6.6(1.9) \%$, respectively; $P=0.26$. In this subgroup, there was a strong positive correlation between $25(\mathrm{OH}) \mathrm{D}$ and the omega-3 index during both seasons. For the total population the Pearson's correlation coefficient was $0.358, P<0.001$, during the winter-spring season and 0.199 , 


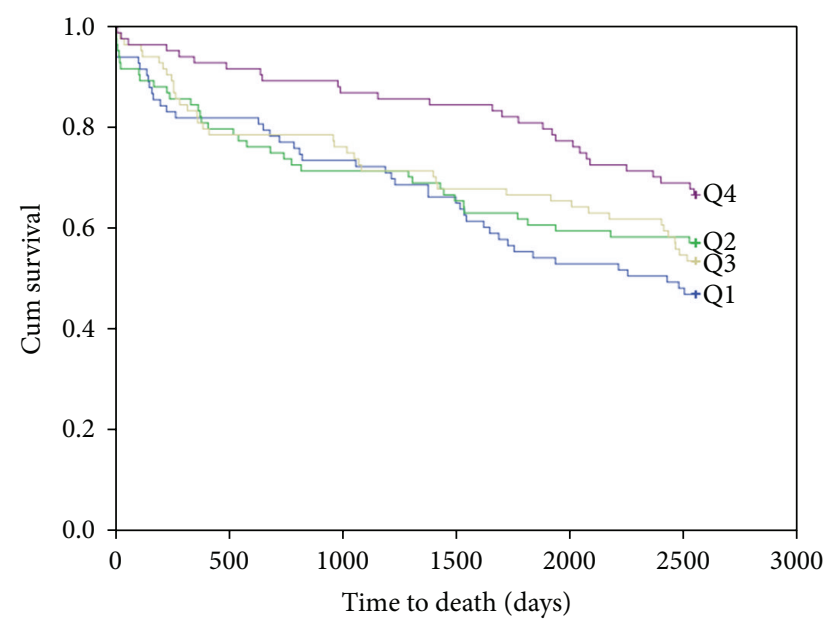

FIGURE 3: Kaplan-Meier plots for seven-year total mortality of $25(\mathrm{OH}) \mathrm{D}$ quartiles in females.

$P=0.006$, during the summer-autumn period, respectively. The Pearson's seasonal correlation coefficient for females was $0.380, P<0.001$ and $0.183 P=0.16$, respectively, and for males $0.347, P<0.001$ and $0.205, P=0.022$, respectively.

\section{Discussion}

Our chest pain population was recruited from the southwestern coast of Norway, at a latitude of $58^{\circ} \mathrm{N}$. At this latitude, the production of cutaneous vitamin $\mathrm{D}$ is low in the winter months during which vitamin $\mathrm{D}$ levels depend on other sources, mainly fish intake.

We divided the population into quartiles of vitamin $\mathrm{D}$ levels, comparing the higher quartiles to the lowest quartile, and performed univariate and multivariable analyses of mortality at 2- and 7-year follow-up.

In the univariate quartile analysis at 2-year follow-up of the total and TnT positive patient population we observed a trend towards decreased total and cardiac mortality in the upper as compared to the lower quartile of vitamin D. These trends approached significant values when applying continuous vitamin D data in the univariate analysis (Table 5). In the female population, the reduction in total and cardiac mortality reached statistical significance by both quartile and continuous univariate analysis.

At 7-year follow-up, both statistical approaches yielded significant results in the univariate analysis, with reduced total and cardiac mortality associated with increasing vitamin D levels in the total and TnT positive population, as well as in the female population, respectively. These and other 7-year follow-up data are shown in Table 5.

These associations were not present after adjusting for confounding variables in our multivariable model. In contrast to studies in which multivariable models have demonstrated a U-shape or reverse J-shape mortality curve across quintiles [4] and quartiles [5] of vitamin D, the statistically nonsignificant results in our multivariable quartile analysis precluded a similar evaluation. However, the univariate quartile hazard ratios in our ACS population showed a similar pattern to that of the above studies $[4,5]$, in which subjects were enrolled from a general elderly population, with the highest age ( $>75$ years) in the study by Jia et al. [4] as compared to $69.6 \pm 14.4$ years in our study. In general, the vitamin D values in our patients were higher than in the two other studies, with the lowest quartile in our study comparable to that of second and third quintiles in the study by Jia et al. [4] and to the second quartile in the study by Visser et al. [5]. Furthermore, less interquartile differences in morbidity and mortality in our study may be related to a tighter range of vitamin D values.

In a previous report from our group we obtained more definitive results investigating a population in Northern Argentina located at a latitude of $24^{\circ}$ and at an altitude more than $1000 \mathrm{~m}$ above sea level. In the latter population, the prognostic utility of vitamin $\mathrm{D}$ in the female population remained highly significant after correcting for confounding factors, which statistically may be due to a larger proportion of females and more events in this subgroup. Similar results have been obtained in a German study by Karakas et al. [22]. However, in that study they included 1783 healthy middleaged subjects of both gender with a follow-up of eleven years during which they identified 298 combined CHD cases, whereas we investigated total and cardiac death in chest pain patients with suspected ACS.

In our Norwegian as compared to our Argentinean study [16] levels of vitamin D were similar in the lowest quartile $(30.5 \pm 5.9 \mathrm{nM}$ versus $30.7 \pm 5.6 \mathrm{nM})$ of the total populations. Levels were also similar in Q2 and Q3, but higher in Q4 in the Norwegian population $(78.6 \pm 13.2 \mathrm{nM}$ versus $72.9 \pm 11.1 \mathrm{nM})$. This was an unexpected finding, as the geographic locations are different with respect to sun exposure.

We have previously [23] published data suggesting that vitamin D status may be related to socioeconomic factors, implying that low vitamin $\mathrm{D}$ levels in that setting may behave as a secondary risk marker. In our present population the quartile levels of vitamin D were clearly related to the difference in dietary capture of vitamin $\mathrm{D}$, as demonstrated by a monotonic increase in omega-3 values from lowest to highest quartile of vitamin $\mathrm{D}$, as shown in Table 2.

Based on our observational data, reduced vitamin D levels may not only be regarded as a marker of morbidity in women as compared to men but may also reflect a gender difference in serum vitamin $\mathrm{D}$ concentration. The mechanism for this difference is not obvious, although a female hormonal interaction has been noted, with levels of vitamin D and progesterone/estrogen being inversely correlated [24].

Optimal levels of 25(OH)D are still under debate. According to Ross et al. [20], levels of at least $50 \mathrm{nM}$ will meet the needs of at least $97.5 \%$ of the population, whereas the median vitamin $\mathrm{D}$ concentration in the general population will be above $40 \mathrm{nM}$. In the present study, we have investigated the significance of vitamin $\mathrm{D}$ levels $<40 \mathrm{nM}$ in relation to outcome. Below $40 \mathrm{nM}(16 \mathrm{ng} / \mathrm{mL})$, vitamin D behaved as an independent predictor for total death both in the univariate and multivariable analysis. As vitamin D deficiency was found to be an independent risk factor at levels below $40 \mathrm{nM}$, higher levels may reflect an overall healthier lifestyle and may not in themselves be causally related to outcome. 
In a subpopulation consisting of TnT positive patients, the correlation between vitamin $\mathrm{D}$ and the omega-3 index, including EPA and DHA, was higher during winter-spring $(r=0.358, P<0.001)$ as compared to the summer-autumn season $(r=0.199, P=0.006)$. As the omega-3 index was similar in both seasons, this finding may be explained by an increased utilization of vitamin $\mathrm{D}$ in the diet during the winter season, and the lower correlation during the summer season may be due to the increased levels of cutaneous vitamin $\mathrm{D}$ production. Our results are in accordance with a recent publication in which the authors compared levels of vitamin D obtained from sun exposure versus diet at the same altitude as in the present study [25].

The association between vitamin $\mathrm{D}$ and omega-3, especially during the winter-spring season, is of interest, as the diet may be the main source of vitamin $\mathrm{D}$ during this period. Furthermore, the correlation was strongest in women in whom vitamin $\mathrm{D}$ showed less seasonal variation. Thus, due to its prognostic implication, vitamin $\mathrm{D}$ may be involved in the beneficial effects of fish intake. Therefore, the natural presence of vitamin D in dietary studies, such as in DART [26] and in the long-term follow-up study by Kromhout et al. [27], may have beneficially affected outcome in these studies, as reflected by the stepwise increase in the omega-3 index from lowest to highest quartile of vitamin $\mathrm{D}$ in the present study. Although reduction in mortality was noted in the omega-3 arm of the GISSI studies [28, 29], some of the other megatrials also applying a purified omega- 3 compound have not shown a reduction in mortality, as noted in a metaanalysis by Kwak et al. [30], involving 20485 patients with a history of cardiovascular disease from 14 randomized, double blind, and placebo controlled studies.

The addition of vitamin D to a purified omega-3 compound is being investigated in the VITAL study (ClinicalTrials.gov Identifier: NCT01169259).

Strengths and Limitations. In comparison to other observational studies enrolling subjects from a general population, the novelty in this study is related to (1) selection of a chest pain population with suspected ACS, (2) blood samples obtained at hospital admission, and (3) categorization of subjects with and without myocardial injury. In contrast to many other studies, we have focused not only on total mortality but also on cardiac death in our selected high risk population. The strength of our study is mainly related to the long-term follow-up and the proportion of females accounting for $39 \%$ of the patients. Furthermore, its design is similar to that of our previously published Argentinean report based on admitted chest pain patients with suspected ACS [16], enabling a comparison of western lifestyle and traditions to that of a South-American population with other traditions and dietary habits. A larger number of patients may have contributed to more consistent findings in our Argentinean population. However, as power calculations are not applicable in observational studies, this should be regarded as a limitation. Also, we did not correct for parathyroid hormone $(\mathrm{PTH})$, but we do not regard this to be of great importance, as previous data would indicate that only very low values of vitamin D will influence the level of PTH [31].
As this is an observational study, unknown confounders may have been missed.

In conclusion, at long-term follow-up the univariate analysis demonstrated a statistically significant lower mortality in the highest as compared to the lowest quartile of vitamin D in chest pain patients with suspected ACS, especially in females. Vitamin D was independently related to mortality at levels below the population median. An increase in the omega-3 index was noted from lowest to highest quartile of vitamin $\mathrm{D}$ and there was a stronger correlation between vitamin $\mathrm{D}$ and the omega-3 index during winter-spring as compared to the summer-autumn season.

\section{Conflict of Interests}

The analyses of the omega-3 index were performed by William S. Harris who is the President of OmegaQuant Analytics, LLC, and a Senior Research Scientist at Health Diagnostic Laboratory, Inc. Both of these laboratories offer omega-3 index testing, the former for researchers/consumers and the latter for clinicians. He is also a consultant to Omthera Pharmaceuticals and Aker Biomarine, companies with interests in omega-3 fatty acids.

\section{References}

[1] V. Kunadian, G. A. Ford, B. Bawamia, W. Qiu, and J. E. Manson, "Vitamin D deficiency and coronary artery disease: a review of the evidence," American Heart Journal, vol. 167, no. 3, pp. 283291, 2014.

[2] P. E. Marik and J. Varon, "Omega-3 dietary supplements and the risk of cardiovascular events: a systematic review," Clinical Cardiology, vol. 32, no. 7, pp. 365-372, 2009.

[3] K. Kienreich, A. Tomaschitz, N. Verheyen et al., "Vitamin D and cardiovascular disease," Nutrients, vol. 5, no. 8, pp. 3005-3021, 2013.

[4] X. Jia, L. S. Aucott, and G. McNeill, "Nutritional status and subsequent all-cause mortality in men and women aged 75 years or over living in the community," British Journal of Nutrition, vol. 98, no. 3, pp. 593-599, 2007.

[5] M. Visser, D. J. H. Deeg, M. T. E. Puts, J. C. Seidell, and P. Lips, "Low serum concentrations of 25-hydroxyvitamin D in older persons and the risk of nursing home admission," The American Journal of Clinical Nutrition, vol. 84, no. 3, pp. 616-622, 2006.

[6] T. J. Wang, M. J. Pencina, S. L. Booth et al., "Vitamin D deficiency and risk of cardiovascular disease," Circulation, vol. 117, no. 4, pp. 503-511, 2008.

[7] H. Aarsetoey, H. Grundt, O. Nygaard, and D. W. T. Nilsen, “The role of long-chained marine N-3 polyunsaturated fatty acids in cardiovascular disease," Cardiology Research and Practice, vol. 2012, Article ID 303456, 15 pages, 2012.

[8] D. Mozaffarian and J. H. Y. Wu, "Omega-3 fatty acids and cardiovascular disease: effects on risk factors, molecular pathways, and clinical events," Journal of the American College of Cardiology, vol. 58, no. 20, pp. 2047-2067, 2011.

[9] W. S. Harris and C. von Schacky, "The Omega-3 Index: a new risk factor for death from coronary heart disease?" Preventive Medicine, vol. 39, no. 1, pp. 212-220, 2004.

[10] M. F. Holick, "Vitamin D: evolutionary, physiological and health perspectives," Current Drug Targets, vol. 12, no. 1, pp. 4-18, 2011. 
[11] A. Mithal, D. A. Wahl, J.-P. Bonjour et al., "Global vitamin D status and determinants of hypovitaminosis D," Osteoporosis International, vol. 20, no. 11, pp. 1807-1820, 2009.

[12] M. F. Holick, "Vitamin D: a D-lightful solution for health," Journal of Investigative Medicine, vol. 59, no. 6, pp. 872-880, 2011.

[13] T. J. Wang, M. J. Pencina, S. L. Booth et al., "Vitamin D deficiency and risk of cardiovascular disease," Circulation, vol. 117, no. 4, pp. 503-511, 2008.

[14] H. Dobnig, S. Pilz, H. Scharnagl et al., "Independent association of low serum 25-hydroxyvitamin D and 1,25-dihydroxyvitamin D levels with all-cause and cardiovascular mortality," Archives of Internal Medicine, vol. 168, no. 12, pp. 1340-1349, 2008.

[15] S. Pilz, W. März, B. Wellnitz et al., "Association of vitamin $\mathrm{D}$ deficiency with heart failure and sudden cardiac death in a large cross-sectional study of patients referred for coronary angiography," Journal of Clinical Endocrinology and Metabolism, vol. 93, no. 10, pp. 3927-3935, 2008.

[16] P. A. Naesgaard, R. A. León de la Fuente, S. T. Nilsen et al., "Serum $25(\mathrm{OH}) \mathrm{D}$ is a 2-year predictor of all-cause mortality, cardiac death and sudden cardiac death in chest pain patients from Northern Argentina," PLoS ONE, vol. 7, no. 9, Article ID e43228, 2012.

[17] P. A. Naesgaard, R. A. L. de la Fuente, S. T. Nilsen et al., "Vitamin $\mathrm{D}$ predicts all-cause and cardiac mortality in females with suspected acute coronary syndrome: a comparison with brain natriuretic peptide and high-sensitivity c-reactive protein," Cardiology Research and Practice, vol. 2013, Article ID 398034, 8 pages, 2013.

[18] V. Pönitz, T. Brügger-Andersen, D. Pritchard, H. Grundt, H. Staines, and D. W. T. Nilsen, "Activated factor XII type A predicts long-term mortality in patients admitted with chest pain," Journal of Thrombosis and Haemostasis, vol. 7, no. 2, pp. 277-287, 2009.

[19] H. Aarsetoey, V. Pönitz, H. Grundt, H. Staines, W. S. Harris, and D. W. T. Nilsen, "(n-3) fatty acid content of red blood cells does not predict risk of future cardiovascular events following an acute coronary syndrome," The Journal of Nutrition, vol. 139, no. 3, pp. 507-513, 2009.

[20] A. C. Ross, J. E. Manson, S. A. Abrams et al., "The 2011 report on dietary reference intakes for calcium and vitamin $\mathrm{D}$ from the Institute of Medicine: what clinicians need to know," Journal of Clinical Endocrinology and Metabolism, vol. 96, no. 1, pp. 53-58, 2011.

[21] M. Müller-Bardorff, K. Hallermayer, A. Schröder et al., "Improved troponin T ELISA specific for cardiac troponin $\mathrm{T}$ isoform: assay development and analytical and clinical validation," Clinical Chemistry, vol. 43, no. 3, pp. 458-466, 1997.

[22] M. Karakas, B. Thorand, A. Zierer et al., "Low levels of serum 25-hydroxyvitamin $\mathrm{D}$ are associated with increased risk of myocardial infarction, especially in women: results from the MONICA/KORA Augsburg case-cohort study," The Journal of Clinical Endocrinology and Metabolism, vol. 98, no. 1, pp. 272280, 2013.

[23] R. A. León de la Fuente, P. A. Naesgaard, S. T. Nilsen et al., "Socioeconomic assessment and impact of social security on outcome in patients admitted with suspected coronary chest pain in the city of Salta, Argentina," Cardiology Research and Practice, vol. 2013, Article ID 807249, 9 pages, 2013.

[24] J. A. Knight, J. Wong, K. M. Blackmore, J. M. Raboud, and R. Vieth, "Vitamin D association with estradiol and progesterone in young women," Cancer Causes and Control, vol. 21, no. 3, pp. 479-483, 2010.
[25] Z. Lagunova, A. C. Porojnicu, L. Aksnes et al., "Effect of vitamin D supplementation and ultraviolet B exposure on serum 25hydroxyvitamin D concentrations in healthy volunteers: a randomized, crossover clinical trial," British Journal of Dermatology, vol. 169, no. 2, pp. 434-440, 2013.

[26] M. L. Burr, A. M. Fehily, J. F. Gilbert et al., "Effects of changes in fat, fish, and fibre intakes on death and myocardial reinfarction: diet and reinfarction trial (DART)," The Lancet, vol. 2, no. 8666, pp. 757-761, 1989.

[27] D. Kromhout, E. B. Bosschieter, and C. De Lezenne Coulander, "The inverse relation between fish consumption and 20-year mortality from coronary heart disease," The New England Journal of Medicine, vol. 312, no. 19, pp. 1205-1209, 1985.

[28] R. Marchioli, "Dietary supplementation with N-3 polyunsaturated fatty acids and vitamin $\mathrm{E}$ after myocardial infarction: results of the GISSI-Prevenzione trial," The Lancet, vol. 354, no. 9177, pp. 447-455, 1999.

[29] GISSI-HF Investigators, "Effect of n-3 polyunsaturated fatty acids in patients with chronic heart failure (the GISSI-HF trial): a randomised, double-blind, placebo-controlled trial," The Lancet, vol. 372, no. 9645, pp. 1223-1230, 2008.

[30] S. M. Kwak, S.-K. Myung, Y. J. Lee, and H. G. Seo, "Efficacy of omega-3 fatty acid supplements (eicosapentaenoic acid and docosahexaenoic acid) in the secondary prevention of cardiovascular disease: a meta-analysis of randomized, double-blind, placebo-controlled trials," Archives of Internal Medicine, vol.172, no. 9, pp. 686-694, 2012.

[31] L. Steingrimsdottir, O. Gunnarsson, O. S. Indridason, L. Franzson, and G. Sigurdsson, "Relationship between serum parathyroid hormone levels, vitamin D sufficiency, and calcium intake," Journal of the American Medical Association, vol. 294, no. 18, pp. 2336-2341, 2005. 


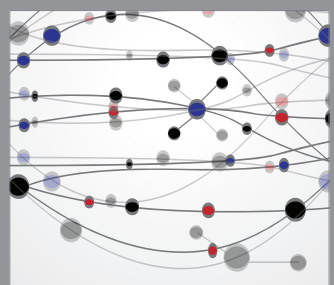

The Scientific World Journal
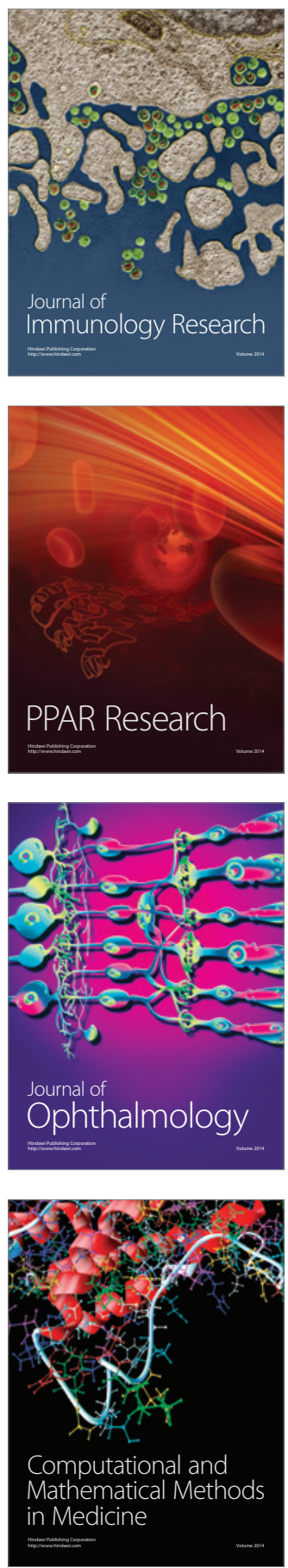

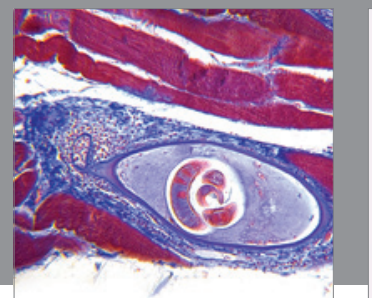

Gastroenterology

Research and Practice
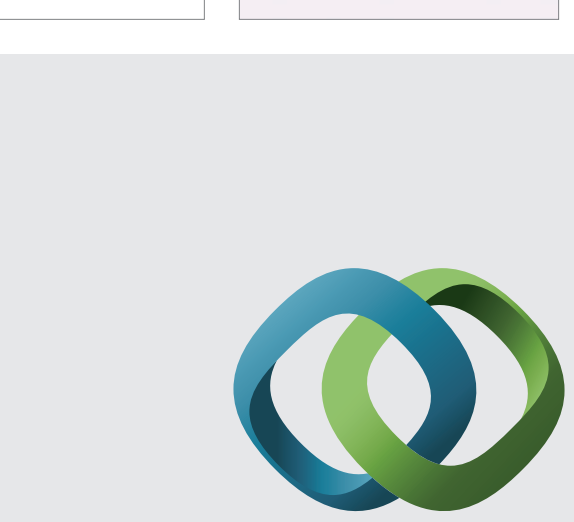

\section{Hindawi}

Submit your manuscripts at

http://www.hindawi.com
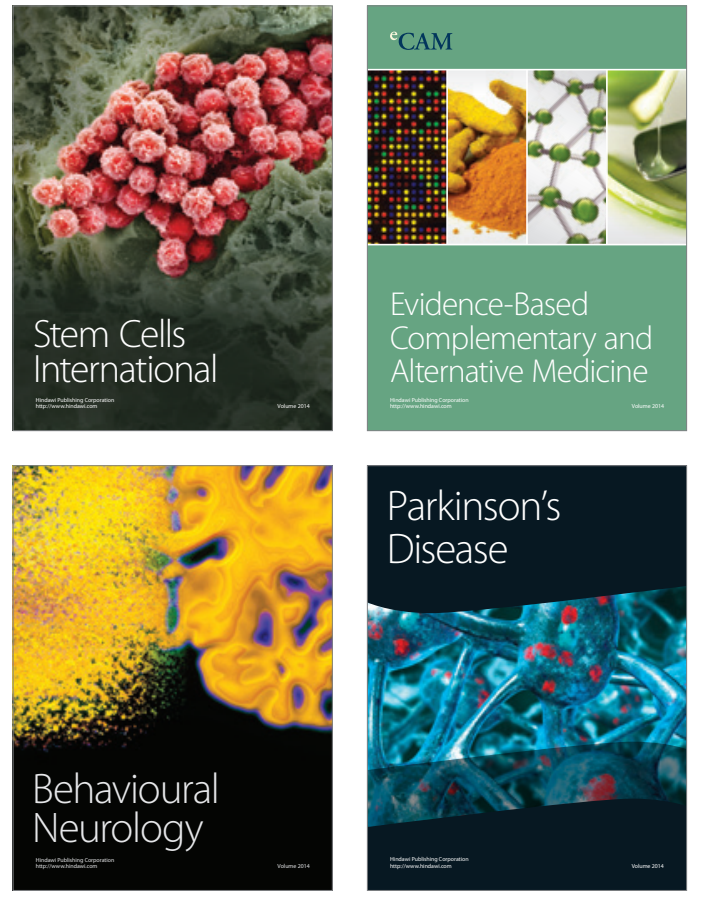
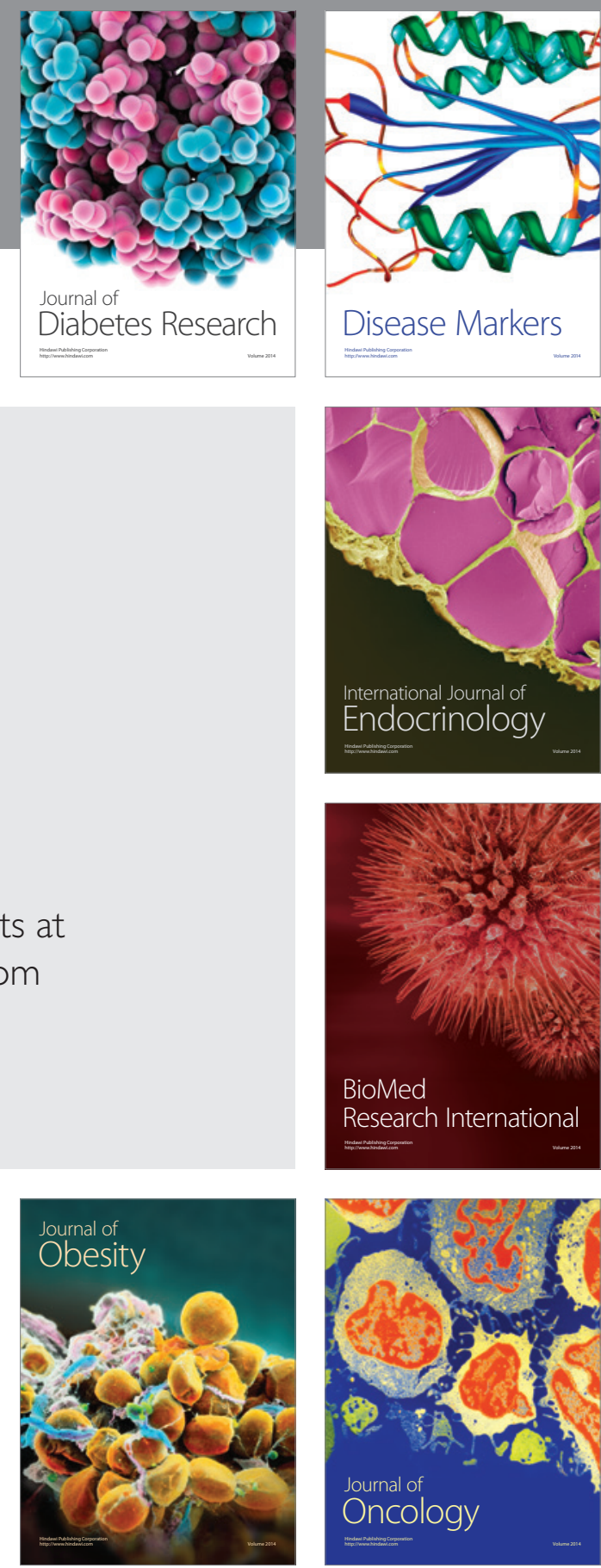

Disease Markers
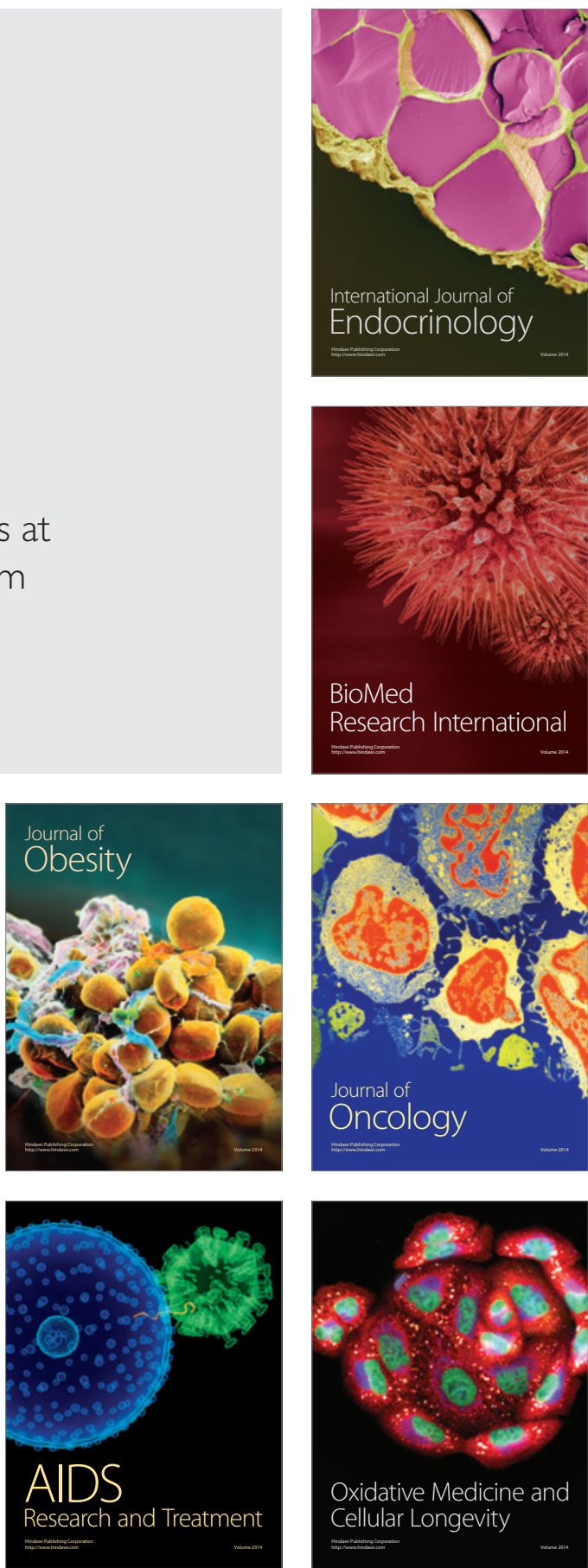